\title{
Article
}

\section{'Multi-directional management': Exploring the challenges of performance in the World Class Programme environment}

Collins, D., and Cruickshank, A.

Available at http://clok.uclan.ac.uk/9437/

Collins, D., ORCID: 0000-0002-7601-0454 and Cruickshank, A. ORCID: 00000002-8893-2341 (2012) 'Multi-directional management': Exploring the challenges of performance in the World Class Programme environment. Reflective Practice, 13 (3). pp. 455-469. ISSN 1462-3943

It is advisable to refer to the publisher's version if you intend to cite from the work. http://dx.doi.org/10.1080/14623943.2012.670630

For more information about UCLan's research in this area go to http://www.uclan.ac.uk/researchgroups/ and search for <name of research Group>.

For information about Research generally at UCLan please go to http://www.uclan.ac.uk/research/

All outputs in CLoK are protected by Intellectual Property Rights law, including Copyright law. Copyright, IPR and Moral Rights for the works on this site are retained by the individual authors and/or other copyright owners. Terms and conditions for use of this material are defined in the policies page. 
This is a pre-proof correct manuscript, as accepted for publication, of an article published by Taylor \& Francis in Reflective Practice on $3^{\text {rd }}$ April 2012, available online:

http://www.tandfonline.com/doi/full/10.1080/14623943.2012.670630\#abstract

PLEASE REFER TO THE PUBLISHED VERSION FOR CITING PURPOSES

"Multidirectional Management": Exploring the challenges of performance in the World

Class Performance environment

Dave Collins \& Andrew Cruickshank

Institute of Coaching and Performance, University of Central Lancashire 


\section{Abstract}

Driven by the ever-increasing intensity of Olympic competition and the "no compromise - no stone unturned' requirements frequently addressed by HM Government and its main agency, UK Sport, a change in culture across Olympic team landscapes is a common occurrence. The main 'change agent', or at least 'change instigator' in UK World Class Programmes is seen as the Performance Director (PD) and recent investigation has focused on unearthing areas of best practice in the role (Fletcher \& Arnold, 2011). However, recognising the complex, social challenge of developing and sustaining a high performing culture and the growing rate of elite team management turnover (cf. Cruickshank \& Collins, in press $\left.^{b}\right)$, there exists an urgent need to consider the context-specific delivery of such pan-individual change; especially as this has played a major role in the realisation of the Olympic Dream, or perhaps Nightmare. With a focus on process, this contribution consequently presents reflections from 8 current or recently serving PDs in the UK system on their experiences of creating and disseminating their vision for their sport, a vital initial activity of the change initiative. To facilitate a broad overview of this construct, reflections are structured around the vision's characteristics and foundations, how it is delivered to key stakeholder groups, how it is influenced by these groups, the qualities required to ensure its longevity and its limitations. Emerging from these perceptions, the creation and maintenance of a shared team vision was portrayed as a highly dynamic task requiring the active management of a number of key internal and external stakeholders. Furthermore, the application of 'dark' traits and context-specific expertise were considered critical attributes for the activity's success. Finally, recent calls for research to elucidate the wider culture optimisation process are reinforced.

Keywords: culture change, dark traits, elite sport, expertise, UK Sport, vision 
As Olympic performance continues to push the boundaries of physical, technical, tactical and mental aptitude, it is crucial that athletes and their support staff operate within high performing cultures throughout the four year competition cycle. Indeed, reflecting the energy, engagement and focus required to improve/refine all facets of performance, and ensure that these are robust to the intense pressure an Olympic Games, a minute-to-minute, second-to-second commitment to one's profession across this period is essential if peak success is to be achieved. As those responsible for managing and regulating individual, intragroup and inter-group features of the performance environment, the perception, decision making and action of the Performance Director (hereafter PD) is therefore central to the functioning, longevity and achievement of Olympic sports teams.

Certainly, while Olympic sport PDs typically deliver little if any 'hands on' coaching (or at least should if their role is not to become 'clouded'), their ability to influence panindividual performance (positively, negatively or indifferently) is arguably the most significant of any in the performance department of a national sport organisation (i.e., that including team management, performance-specific administrative staff, support staff and performers) apart from the personal coach him/herself. Specifically, through holding ultimate responsibility for team policies, systems, structures and processes, at least in an optimum system, a PD's ambitions, principles and intentions will always, to at least some extent, be reflected in the perceptions, preferences and behaviours of all those 'lower down' the organisational chart (we place lower down in inverted commas as this principle applies to democratic as well as autocratic models). As such, due to growing awareness of this role's significance, attention has recently turned to consider these figures perceptions of best practice.

Specifically, from enquiry into the position's main facets and the qualities required for optimal performance, Fletcher and Arnold (2011, p.223) revealed four areas of Olympic 
PD competency, namely: operations, people, culture and vision. Operations entailed "financial management, strategic competition and training planning, athlete selection for competition, and upholding rules and regulations"; people involved "staff management, lines of communication, and feedback mechanisms"; culture was represented by "establishing role awareness, and organizational and team atmosphere"; and, finally, vision, or "the team's ultimate aspiration" (p. 228) incorporated "vision development, influences on the vision, and sharing the vision". Providing much needed insight into the nuances of Olympic team management, this work offers a valuable overview of the required dimensions of proficiency, albeit that the competency construct may impose some limitations on our conceptual understanding of how exactly such 'competencies' are differentially blended and applied (cf. in coaching: Abraham \& Collins, 2011; in support science: Martindale \& Collins, 2007). Indeed, as alluded to by Fletcher and Arnold, it is reasonable to assert that the relative importance and operationalisation of each identified theme will vary substantially across contextually distinct phases and episodes of the real life PD challenge.

Expressly, while many of the sub-theme responsibilities identified by Fletcher and Arnold (2011) represent inherent and ongoing tests of the PD skill set, successful engagement in each area will undoubtedly be highly context-specific. For example, as in any complex and dynamic environment, factors such as history, tradition, systems, structures and interpersonal relationships will all interact to dictate/limit the options and directions available to the PD in their efforts to deliver sustained optimal performance (Bevir \& Richards, 2009; Cilliers, 2000). Accordingly, the uncritical, pan-context application of generic 'advantageous' leadership behaviours, such as those espoused by transformational leadership theory (cf. Callow, Smith, Hardy, Arthur, \& Hardy, 2009), across a variety of scenarios will presumably often generate sub-optimal impact or, in some instances, even be inappropriate. 
Of further importance, some of the Olympic PD's broad activities also appear highly context-dependent. Specifically, the efficient and effective creation of a shared vision and culture will logically only occur if pertinent groups (i.e., team management; administrators; support staff; performers) and their members consider that: a) the old vision/culture is no longer engaging and/or functional; or b) the new vision/culture holds greater rewards and/or is more appealing. Normally generated (or demanded) by (usually) tacitly accepted watershed moments, the Olympic Games' four-year schedule provides one such 'natural' tipping point (Kim \& Mauborgne, 2003) whereby a detailed review of performance provides a catalyst for determining the focus and approach in the next cycle. Beyond this structural feature, however, one exemplar 'unnatural' trigger is that of PD turnover. In this scenario a new, incoming director engages in a 'change management' programme aimed at fostering a high performing culture and, by association, consistent high performance (cf. Cruickshank \& Collins, in press ${ }^{\mathrm{a}}$ ). Reflecting the sacking pandemic engulfing elite sport systems around the world (Bruinshoofd \& ter Weel, 2003; League Managers Association, 2010; Zinser, 2008) and the lack of peer-reviewed knowledge on this performance-defining construct, our contribution is located within this latter context; in short, how may the benefits of such change be optimised, albeit from the perspective of the incoming director.

Returning to the competencies identified by Fletcher and Arnold (2011), but with a focus on the process of a new director's programme, we place the creation and dissemination of the team's vision as the chronological origins of a change imitative. Indeed, these authors' also conceptualised the Olympic PD's vision as a framework against which individual, team and organisational performance/process goals are harnessed, a point echoed by one of the PDs (individual sport) interviewed for the present contribution:

Having a vision is first and the vision is within you...The next stage only makes a difference if the other ones are in first.....People think attention to detail is 
compelling and necessary, which it probably is, but the environment and the attitude is way more compelling....I can...start introducing [performance] analysis and even physiological testing, but if we don't have a compelling performance environment that stuff's just a waste of time. In fact it's more than a waste of time, it's a distraction.

Recognising the programme- and outcome-shaping nature of the guiding vision, examining PDs' reflections on this construct is therefore both theoretically and practically intriguing. Beyond contributing to the developing literature and evidence-based practice of managementlead culture change elite sport performance teams (cf. Cruickshank \& Collins, in press ${ }^{\mathrm{a}}$; Cruickshank \& Collins, in press $^{\text {b }}$, we hope that such exposure will stimulate further reflection on the part of elite team managers, consultants who support their programmes and other pertinent members of elite sport organisations (e.g., CEO's; Board members).

Accordingly, to provide a descriptive account of the characteristics, components, interactions and limitations of the PD-facilitated team vision and examine these figures' role in optimising performance, reflections are structured around the following questions: What are the characteristics and foundations of Olympic PDs' visions? How are these best delivered to key stakeholder groups? How are such visions influenced by these groups? What qualities are required to ensure their longevity? And finally, what are their limitations? Mirroring the complexity of elite team environments (cf. Cruickshank \& Collins, in press ${ }^{\mathrm{a}}$ ), we pay close attention to interactional elements, considering how PD decision making, action and reaction does not operate within an uncontested vacuum. The presented reflections come from interviews with eight PDs either currently or previously employed by British Olympic sport organisations within the past two Olympic cycles (2004-2012) (cf. Cruickshank \& Collins, 2011).

\section{Characteristics and foundations of the Olympic PD's vision}


As governed and reinforced by their continued operation within elite sport settings, all PDs articulated that their vision for their sport represented an enduring pursuit for/realisation of optimal performance, which in all cases was directly related to the attainment of desirable objective outcomes (i.e., medals/titles). Resonating with a number of acquired perceptions, the following quote conveys how one individual's vision (individual sport) was built upon a foundation of ambition, enticing possibility and positive engagement:

Very simply...[the vision] was to produce or develop the best sports team in Britain and the best [sport] team in the World. And it's a very grand, broad statement obviously, backed up by the imagery it creates in people...They're dominant forces in whatever it is that they're contesting...it's a tidal wave, an unstoppable force...there will be a way in which they race or play...We wanted to race exciting, attacking races. We wanted to win races, not cross the line first by default having buried everyone into submission. We wanted to actually go win 'em.

Interestingly, while all interviewees held similar ideals regarding their long-term hopes and goals, it was also acknowledged, particularly by the PD quoted immediately above, that such aspiration was a mandatory but initially mysterious and elusive construct:

Did I know the detail of what I was trying to achieve at the time? No. But you have this vision and I think perhaps that's actually worth stating: When you make that pledge [about] what...you [are] trying to achieve [to key stakeholders], you've got to have a compelling vision....And that pretty much bound [the strategic plan], we're gonna be the best sports team in the World...you want to get on board with that? Come on. What does it actually mean? Don't really know in all detail. But it's gonna be fun and it's gonna be dominant.

From this quote, ambition and optimism were vital components of this PD's initial efforts to immediately engage key stakeholders in their change programme. Importantly, although this and all other PD visions were largely ego-orientated (i.e., they involved winning events 
and/or comparison with others), it is also critical to note that all interviewees clearly clarified that objective rewards did not necessarily define the success of their programmes. Certainly, a key adjunct to the fundamental 'dream' was an acceptance that performance, and therefore the acquisition of medals/titles, is dictated by an innumerate range of athlete-related (e.g., physiological traits; technical expertise), programme-related (e.g., training facilities; sport science/medicine support) and sport/competition-related (e.g., ability of competitors; rule changes) variables. In line with this recognition, one PD (individual and team sport) who described their intention to change a "culture of being a participant to trying to be a winner" reflected:

[The Olympics are] the biggest meet of your life and if you can have the performance of your life, whether you come twelfth or first, you'll feel like you've won a medal cos you couldn't have done any better - you just had the performance of your life. And that's what I want to create...it doesn't matter where you finish, I just want you to come away and go 'god, I couldn't have done any better'...there is nothing worse than ifs and buts.

Acknowledging the demands placed on Olympic PDs by their staff and performers, employers (i.e., national sports organisations), funders (i.e., UK Sport), external partners (e.g., service providers; sponsors) and the media/general public (particularly in the lead up to London 2012), understanding the personal context of why these individuals undertake such roles represents an further important point of reflection. Consistent across the present sample's perceptions, the personal challenge of realising their vision was a key motivator:

I was driven, I was driven by winning, not being placed but by winning; [I] believed that we could find a way to get the right people to challenge the world's best...I'm a proud person as well, I want to win medals...as I did when I was an athlete so that was a massive driver for me. (team sport PD) 
As suggested by this quote, although Olympic PDs are required to facilitate the performance of a multitude of individuals (athletes, support staff, administrative staff), an important but perhaps often overlooked consideration, both academically and practically, is recognition that elite team managers are performers in their own right. Certainly, acknowledging the inherent competitiveness of elite sport environments and the likelihood that PDs will have previously performed, managed/coached or operated in high pressure sport environments, this oversight is surprising. Notwithstanding this point, however, while personal achievement emerged as central to the energy and commitment devoted to programme delivery, significantly none of the interviewees placed this as their sole or major pursuit. First and foremost, all PDs described a desire to introduce and/or optimise systems, structures and processes which would facilitate sustained peak performance in their athletes/staff and, consequently, provide a legacy for their sport:

I saw this as an unbelievable challenge and I liked that... Let's see if we can do it, let's give it a go, cos I liked that, let's see what happens... [But] I want to build them a sporting system... so that if I'm there 10 years and I leave does it just fall apart and crumble? If it does, that's a pretty poor job I did. If there's something there that helps keep producing medals for 50 years then I've done something right; so that's probably the biggest thing I'm trying to achieve. (individual and team sport PD)

Reflecting the magnitude of these aspirations and the personal stake in programme success, all PDs also ardently reported that a robust and enduring personal belief was critical for optimal engagement with the vision and its effective and efficient proliferation within and across generations of the performance department:

You've got to believe in it - if you don't you will not convince anybody else because it's going to be hard and you've got to compel people to come with you, and you've got to set a tone and it's got to be consistent....Can someone like myself take that same change, that culture development from [sport] into [another sport]? I 
personally believe that you can to an extent, but in the end you are fundamentally limited because you actually don't care about it. There might be bits of it you care about it, you might care about being personally successful or something like that, but ultimately you don't understand a sport or fundamentally care about it. And I think that's limiting because you have to believe very strongly in what you're doing....You're going to be up at 1 or 2 o'clock in the morning. (individual sport PD) The clear and stated existence of this longer term goal, namely the generation and achievement of a legacy (cf. the basis under which the Singapore proposal which secured the games for London was based) stands in contrast to the explicit focus by management structures on this oft cited but insufficiently targeted outcome of the home games!

\section{Making the vision work: Ensuring credibility}

While the foundations of the PDs' visions were purely performance-focused, there was widespread recognition that this aspiration's initial formation and dissemination was highly susceptible to a range of mediating factors. Primarily, whether the PD was from 'within' or 'outside' the sport emerged as central to how the vision was generated, delivered and established. For example, one PD (individual and team sport) with no history or previous association with their sport offered a particularly notable reflection on their 'status' at the time of their appointment:

One of the benefits when you come from outside the sport... is that you are not saddled by the history of the sport,...relationships good or bad with people within the sport...[or] the politics of the sport...As such, coming from outside the sport, the fact that you are unknown can be a double-edged sword: It can make people very defensive but it can also make people very, very open by the fact that you are not weighed down by history.... I think you have to be aware you're only in that unique position for a period of time. 
While this PD valued the opportunity to work with a 'clean slate', as facilitated by their lack of connection, the hurdles facing those attempting to deliver sustainable change in unfamiliar settings was significantly reinforced:

To make change....you have to be perceived as...a guru...or...your arguments have to be [flawless] ... I do think the danger of being 'the guru' is that people can be too accepting of change...the reasoning can be flawed.... Even people that have the perception of being a guru in one sport once they move to another sport [encounter difficulties]...[Sir Clive Woodward] obviously is probably a good example of that...went from rugby to football and had a pretty rough time and has gone to the BOA and probably had an even tougher time... If you have that type of stature, you probably need to be relatively humble about how you use it as well, to throw yourself around as the great I AM, even if you are the great I AM is probably a mistake if you're outside of your own field.

Intriguingly, while the PD's stature may require careful handling if the desired vision is to be widely accepted, shared and driven by all performance team members (the principles of a high performing culture: cf. Cruickshank \& Collins, in press ${ }^{\mathrm{b}}$ ), it was also considered invaluable in gaining support and minimising uncertainty and/or resistance from key stakeholder groups:

I do think you need to have a level of credibility, whatever your background is. I think a precursor for being a PD is to have had, even if it's in different sport, is to have had some success... Credibility does count for an awful, awful lot...you wouldn't be being questioned directly by the people who are going out to perform on your behalf. (individual and team sport PD)

Importantly, while the PD's credibility at appointment emerged as a consistent reflection, it is also essential to note that the credibility of the vision itself also offered a mechanism by which the programme could be protected from derailment by powerful individuals: 
What you're backing is your long term vision to be the best team in the world:

We're not the best team in the world at the minute so we have to move forward, we can't just do what we're doing now. And if I'm right it's going to require you guys getting better, and if I can have some demonstrable evidence that at least some of you, and significant ones of you [are] getting better then there's enough of a hook that when [a socially powerful, senior performer] walks you don't. (individual sport PD)

In cases where such a vision lacked credibility, the role of other agencies (such as CEO,

Board, UK Sport, etc.) was seen as crucial to keeping the plan on track.

Particularly in the early stages of selling the new vision, it's essential to get all the other stakeholders on board and publicly supporting the plan. Without this you are 'a voice which crieth in the wilderness.' (individual and team sport PD)

This reflection notably emphasises the importance of multidirectional management if selling the vision is to be successful, a point which we now consider in greater depth.

\section{Creating and disseminating the vision: Multi-stakeholder influence}

As identified above, although the PD's vision for their sport may be firmly grounded within a philosophy of facilitating enduring optimal performance, the preceding reflections support our earlier assertion that elite sport is a highly dynamic and contested environment which is both rooted to and shaped by fluctuating environmental contexts. Indeed, across the present data set, powerful actors from a variety of stakeholder groups emerged as pivotal in constraining the PD's vision and associated activities. As such, multisource information gathering and multi-stakeholder negotiation, focused on both internal and external parties, emerged as a critical success factor of vision formulation and dissemination.

\section{Influences from within the performance environment}

Highlighting the challenge of delivering a constantly coherent programme, one PD (individual and team sport) reported the importance of gaining the support of existing senior 
managers before fully engaging with the changes which would support the attainment of their vision:

They were very, very cautious... and that somewhat dictated my approach...I knew that I had to develop a relationship with those two [senior managers] in particular. Without a relationship with those two people...then I would have been doomed to fail.

Beyond ensuring that the whole management team exuded a unified and consistent approach, the same PD also reflected upon similar issues within their support staff, often providing a distraction to immediately addressing the dissemination of their vision and pertinent performance-related issues:

[Consider] my good [national] coach who will talk at you. The first meeting I had with him two hours after I started the job he gave me a document which was 2500 pages long to read, knowing full well that I wouldn't read it - he knew that I knew that - but it was a way of him putting a line in the sand and saying don't think you're gonna get the better of me.

Similarly, all PDs also revealed that sensitivity to performers' perceptions was critical in ensuring that the programme's new systems and processes were positively received:

You're asking people to commit way above and beyond what it is ordinarily committed if you're going to be the best in the world, and it's an undefined quantity and it's a scary quantity for most people... If we're going to chop people on programmes every six months, they ain't going to [optimally] commit...They...will do it out of fear. Well now you're not free you're not liberated and in order to truly give you've got to be liberated. The brakes have got to come off, and fear is a break (individual sport PD).

\section{Influences from outside the performance environment}

As well as the constraining perceptions, expectations and actions of performers, support staff and team management, the PDs also reflected upon the influence which external groups have 
on the establishment and diffusion of their team's visions. Predominantly, such assessment related to interactions with UK Sport (the British government's Olympic sport funding body and largest provider of finance to participating teams), the sport's wider membership and the media.

\section{Influences from above}

As suggested, managing interactions with UK Sport was portrayed as a particular challenge to the PD's efforts in establishing and sharing their vision. Primarily, this body's insatiable pursuit of medals provided significant issues for many and their desire to remain sensitive to the internal uncertainty in the performance environment triggered by the PD's impending new programme:

The first role I [had]...was to write the four-year plan to UK Sport...The timing of it worked, personally, particularly poorly...I was keen to keep everybody happy and probably should have just looked at my own needs a little bit more...but we had to very quickly put together a concise summary performance plan as to the changes we were going to make during this cycle...,the significant events we were going to be attending and what our medal targets were for those events...From my point of view... [the plan] was in effect a lip service paper exercise which was required by government, that was not the point in time to be making people very, very defensive about my input. (individual and team sport PD)

In contrast, another PD felt the need to use the planning process as a line in the sand: My first plan was an important one; an exercise in managing upwards aimed more at creating the space necessary to operate against the performance models required than a pure box ticking exercise. I had to do it to keep them off my back! (individual and team sport PD) 
Indeed, rather than ensuring that PDs were liberated to create and deliver their own vision, one interviewee revealed that UK Sport's growing preference for involvement in performance matters caused a further significant distraction:

I don't need to copy a blueprint from British Cycling... Although UK Sport tried to simplify everything, [and] there's definitely good practice that can go across all sports, [they] aren't going to tell me about the nuances of [sport] and what I need to put in place. (team sport PD)

Indeed, although not responsible for preparing athletes for Olympic competition, another PD (individual sport) provided detailed and insightful reflection on UK Sport's often authoritarian involvement and preoccupation with medals as the marker of successful investment:

If UK Sport don't believe in [your] model then we have a problem...I could have gone, yeah, I can see [the merits of UK Sport's ideas]...but fundamentally we're on different pages... Underpinning all this conversation...compelling visions, real belief in what you want to do, absolute immersion and determination to see it through..., if you're putting a lot into it on somebody else's agenda that's really dissatisfying... You have to absolutely believe, personally believe in what you're doing and [problems arise] if it has to be framed by somebody else.

Interestingly, while all PDs aimed to deliver long-term performance optimisation, another interviewee (individual and team sport PD) reflected on how the pressure for medals had ultimately shaped their initial focus and approach at the expense of changes which would facilitate such sustained achievement:

I am answerable to UK Sport at the same time as being answerable to the sport itself so it does become a balance... One of the things I've tried not to do...is try not to get overly involved in changing the governing body per se...that can become a massive distraction... If my tenure was to last for an extended period of time maybe those are some conversations I would have further down the line. At this point in time, I 
suppose I'm fairly cocooned and certainly extremely focused on...performance outcome.

Against this pragmatic focus a number of other influences were apparent. Some of these are considered in the next section.

\section{Influences from the side}

Of final note, interviewees also identified challenges to their vision from influential stakeholders outside of the performance and organisational environment, such as the sport's wider membership:

The membership of the sport runs into the hundreds of thousands and of course the people involved in high performance are just a small fraction of that so of course there will probably be a raft of people who won't know me, won't understand my role... There is always the dilemma in Olympic Sport that high performance tends to be hugely resource-intensive so you spend a lot of cash on a very...small group...it's either public money or membership money. (individual and team sport PD)

Although not direct targets or recipients of the PD's change programme, the above reflection conveys that lower-level competitive and recreational athletes are nonetheless 'active players' in shaping and sustaining the vision and culture of the performance environment. Indeed, inherent within this quote is recognition that individuals will always have an opinion on the activities of their sport, regardless of their personal impact. As such, while not necessarily shaped by this group's views, the PD's vision must nonetheless be delivered in a manner which facilitates their acceptance or, perhaps more likely, minimises potential discontent.

Beyond the sport's wider membership, a number of PDs also highlighted the media as an important change agent. Indeed, one interviewee (individual sport) reflected on how this group's power could be harnessed to facilitate the realisation of their vision:

We're a small programme, we're genuinely a small sport and I don't want to be a small sport - I want to be a big sport, I want to be a profile sport. So if we can get 
that out there then people think [sport] is significant and that's helpful...If we can get a profile piece on [athlete] in national press...media will pick up with [other individual athletes'] agents because [sport] is gaining momentum.

However, as with many of the vision-shaping factors described above, the same PD also reflected on the 'flipside' of media exposure:

On one hand everyone's really grateful because everyone's benefiting. There's more money coming in, more individual sponsorships, everyone likes to see their name in the paper...[But] then you've got to manage the egos...It's a double-edged sword.

In 'higher profile' sports, these two competing pressures often coalesced as ex-athletes fulfilling powerful media roles took on an excessive influence, with funders if not with the general public!

You get some guys on the TV with strong opinions and the ego to express them. The fact that these are often ill-informed and completely self-serving is often missed by the image conscious guys at UK Sport and in government. (individual and team sport PD)

\section{Delivering the vision: Exemplar qualities required by the PD}

Having offered reflection on the origins, drivers and mediators of the Olympic PD's vision, to maintain our focus on process we now examine the management qualities which these individuals have employed to successfully disseminate and protect this goal.

\section{The 'dark side' of elite team management}

As reported by Fletcher and Arnold (2011, p. 237), characterising Olympic PD’s as holders of an array of socially desirable qualities (e.g., conscientiousness, openness, charisma) presents a "somewhat simplistic picture" of effective management in this domain. Indeed, strongly aligning with this point, the PDs interviewed for the present contribution also conveyed the need for 'darker' attributes in the facilitation and propagation of their vision. For example, take one (individual sport) PD's reflection on the need for soft traits: 
When you look at a lot of successful people they bulldoze...because I think taking people with you is about a compelling vision not about a nice world... The bulldozer analogy for me is: we're gonna go that way because it's right... [and] if we're going that way then let's go that way. Does that make people go 'that was a lovely discussion'; well not really. If we know the end point of this discussion lets go to...that end point now. Whether I've said hello and have a nice day to you or not doesn't really matter. Do people then reflect and go, 'he's a very nice guy'? No, not necessarily. Really, it's a very outcome-generated thing.

As expressed by this quote, the possession and utilisation of an intense and no compromise attitude to performance issues emerged as crucial in ensuring that the PD's sport continually strived to address and solve performance-oriented issues. Indeed, such 'ruthlessness' (a word used by several PDs) was considered particularly valuable for vision dissemination and consequently generating and sustaining an entirely performance-focused culture; an arguably 'dark but positive' feature for these change agents. Further resonating with earlier reflections that placed the sport's enduring success ahead of personal achievement, another PD (team sport) also revealed how an unyielding stance was, at times, mandatory:

Ultimately...I made some decisions which made me quite unpopular but I never came into the sport to be popular anyway, I came in to drive things forward and win medals... The bottom line for me is that [for] the support staff who didn't buy into that, [it] didn't make one bit of difference to me if [they] went or not.

Interestingly, and opposed to contemporary leadership theory assertions on the utility of individual consideration (cf. Callow et al., 2009), a similar direct approach was also often employed with performers:

I was keeping as much as I could all the politics away from the other coaches and the players because they didn't need to know anything about that other than this is the four-year pathway..., this is what it looks like on the playing side this is what you will be expected to do: Are you wanting to be part of this or not because I'm not 
here to negotiate how little you can do, this is about Olympic [sport], you need to change your mindset. (team sport PD)

\section{Context-specific expertise}

As noted in the introduction to this contribution, portioning responsibilities of the Olympic PD into generic competencies provides a valuable but ultimately unsophisticated picture of the skills required by such individuals. Indeed, as supported by the preceding reflections, the complexity inherent within elite sport environments demands the ability to make context-sensitive decisions which ensure that a short- and long-term focus on performance is sustained at all times. Reinforcing this key point, one PD (team sport) reflected:

How much change do you make? When do you make that change? When is change like that too close to the major competition to then become disruptive? You're gonna get some backlash because...the [athletes] will definitely have a downturn in performance and they're going to blame that on you...

Similarly, the situation-specific challenge generated by a number of individuals interacting in ever-evolving environmental contexts was also manifest in efforts to engage key stakeholders in more macro-level decisions aimed at facilitating the vision's actualisation:

The process was different for different pieces of work...The selling of the idea...of posts and people was a very different process to the consultancy approach when it came to revising...the policy of how we supported athletes; in the same way as [it was different to]....the process of...being a lot more prescriptive about the processes that were put in place for bringing people onto and taking people off of the WC programme. (individual and team sport PD)

As such, the picture painted by the present sample was one whereby the dynamic, resourcedemanding nature of managing an elite performance department relies on moment-to-moment 
expertise rather than the ability to deliver prescriptive, generic response patterns. Indeed, one PD (team sport) noted how such a top-down approach is rarely viable:

In the heat of the battle...you're making decisions on the hoof...the reality is you aren't that clear in the middle of it but you use instinct and...gut feeling which defines you...Certainly communication with the players was massive.

\section{Limitations of the vision}

Having considered the characteristics, components and interactional elements of Olympic PDs' visions, a final important consideration lies in perceptions of their restrictions; or more simply, what the vision is not. Indeed, although this ultimate aspiration provides a cornerstone against which long-term culture optimisation is consequently delivered, it is significant to note that a number of PDs reflected on the construct's frailties for generating sustained application and high performance throughout the four-year cycle:

The first hook's the compelling vision: Do you want to be a part of this? [However] that can only last so long, that's rather like the motivational speaker that actually gives you nothing tangible....There's probably enough in your background and track record that says 'maybe this guy can do that' and that lasts a little while, but not long. (individual sport PD)

Accordingly, this reflection suggests that any 'honeymoon' period of heightened optimism, motivation and harmony, as driven by a new and fresh impetus, is relatively short-lived. In essence, if any initial wave of excitement is not followed up with continued dissemination and the introduction, optimisation and monitoring of vision-consistent systems, structures and processes, then it runs significant risk of becoming an historical artefact as opposed to a guiding ideology. Significantly, however, context yet again appears to dictate the PD's possibilities for action, as evaluated by the PD (individual and team sport) who earlier reflected on the 'lip service exercise' requirements of UK Sport: 
Whilst the personal timing of my appointment was poor as I came in, the timing of it in relation to giving me the [full four-year] cycle to develop the programme, I can see that as a positive now. So I suppose if I was ever looking in future, I'd probably be quite mindful of when those key milestones were: How much time you have to make change. I think anybody that comes into the role of PD now (June, 2010) in relation to London has a pretty thankless task, certainly for significant underperformers.

Once more, context-specific expertise is clearly necessary if the PD's change management programme is to be successful. Certainly, the practical prudency of applying general competencies to specific, socially-complex events is, at best, sub-optimal.

\section{Lessons learned: Reflections on the key messages}

Having presented a broad overview of the range of factors which may shape the characteristics, components and agency of the Olympic PD's vision, we now conclude with some reflections of our own regarding the principle messages to emerge from this contribution. Recognising that reflective practice facilitates experiential learning, enhances practice-based knowledge and optimises practice itself (Ghaye \& Lillyman, 2000), such a synopsis, we hope, will encourage similar reflection by other support consultants, their PD clients and other relevant members of national sport organisations (e.g., CEO's).

Primarily, as reflected by the coverage above, creating and sustaining a shared vision in Olympic sports teams (at least British Olympic teams) appears to be a highly dynamic and multifaceted task. While we have focused on one initial component of a pan-individual change programme, the complexity inherent within this process alone suggests that top-down approaches to performance optimisation in Olympic sport settings are neither appropriate nor likely to be successful. Indeed, due to the multitude of groups and individuals which have a 'stake' in performance or performance-related matters, it appears that the Olympic PD would 
be wise to actively manage the perceptions, expectations and agency of such parties to ensure that their programmes run as effectively and smoothly as possible.

Accordingly, rather than a unidirectional model of action whereby stakeholder feedback is neither embraced nor encouraged, the PD challenge seems best represented by a multidirectional framework (see Figure 1). Indeed, a failure to engage with this style of management may be a fundamental error when recognising such groups' power to derail a PD's programme through: a) direct, overt resistance (cf. solid arrows in Figure 1); or b) indirect, covert proliferation/reinforcement of contradictory/rogue perceptions and behaviours with fellow stakeholders (cf. broken arrows in Figure 1). Of course, if managed well then such interactional relationships may be harnessed to subtly facilitate and strengthen the PD's intended vision and culture.

\section{*Figure 1 here*}

Of second key note, the emergence of 'dark' practices and context-specific expertise stimulates significant re-evaluation of traditional approaches to sports team leadership. Regarding the former, even though Fletcher and Arnold (2011) highlighted the prevalence of dark qualities in Olympic PDs (e.g., Machiavellianism; dominance), little insight was provided as to their behavioural manifestation. As detailed above, the present PD sample reflected upon the utility of an unwavering and forceful approach to vision-related decision making, often at the expense of socially desirable action. Indeed, this appeared a conscious approach with many, which is perhaps unsurprising when considering these figures drive for success and the pressure they are under to deliver medals for outcome-focused funders.

Additionally, and in direct contrast to competency-based models of performance leadership, the emergence of context-specific expertise as a critical marker of successfully creating and disseminating the team's vision is significant. Arguably driven by the postpositivist facilitation of global leadership models (e.g., transformational leadership: cf. 
Callow et al., 2009), knowledge on how team managers select, deploy and monitor their behaviour in precise situations is largely unknown. Although this paper has focused on just one feature of Olympic team direction, the reflections offered here suggest that the ability to efficiently compare and contrast the utility of disparate courses of action against the demands of the specific scenario and long term cultural ideals is critical.

Finally, as evidenced by the PDs' perceptions of their vision's limitations, the call for research on the culture optimisation process is significantly reinforced (cf. Fletcher \& Arnold, 2011; Cruickshank \& Collins, in press ${ }^{\mathrm{a}}$; Cruickshank \& Collins, in press ${ }^{\mathrm{b}}$ ). Indeed, recognising that the PD's vision may be an initially necessary but consequently transient feature of a long-term programme, the need for a framework which supports the effective and efficient delivery of a high performing culture is crucial for both the consistency of the Olympic sports team's performance and the long-term career prospects of the PD!

\section{Notes on contributors}

Dave Collins is Professor of Coaching and Performance and Director of the Institute of Coaching and Performance at the University of Central Lancashire. As a long term researchpractitioner in the promotion of performance, Dave's work has increasingly focused on the crucial role played by management systems and organisational environments. As

Performance director of UK Athletics, Dave 'enjoyed' the practical challenges and pressures inherent in the change management process.

Andrew Cruickshank is a $\mathrm{PhD}$ student within the University of Central Lancashire's Institute of Coaching and Performance, examining management-lead culture change in elite sport performance teams. Formerly a professional footballer, Andrew is also completing his final 
stages of training in becoming a British Psychological Society-chartered sport and exercise psychologist.

\section{References}

Abraham, A., \& Collins, D. (in press). Taking the next step: New directions for coaching science. Quest.

Bevir, M., \& Richards, D. (2009). Decentring policy networks: A theoretical agenda. Public Administration, 87, 3-14.

Bruinshoofd, A., \& ter Weel, B. (2003). Manager to go? Performance dips reconsidered with evidence from Dutch football. European Journal of Operational Research, 148, 233246. doi: 10.1016/S0377-2217(02)00680-X

Callow, N., Smith, M.J., Hardy, L., Arthur, C.A., \& Hardy, J. (2009). Measurement of transformational leadership and its relationship with team cohesion and performance level. Journal of Applied Sport Psychology, 21, 395-412. doi:

$10.1080 / 10413200903204754$

Cilliers, P. (2000) What can we learn from a theory of complexity? Emergence, 2(1), pp. 2333.

Cruickshank, A., \& Collins, D., (in press ${ }^{\mathrm{a}}$ ). Change management: The case of the elite sport performance team. Journal of Change Management.

Cruickshank, A., \& Collins, D., (in press ${ }^{\mathrm{b}}$ ). Culture change in elite sport performance teams: Examining and Advancing Effectiveness in the New Era. Journal of Applied Sport Psychology.

Cruickshank, A., \& Collins, D. (2011). Manuscript in preparation.

Fletcher, D., \& Arnold, R. (2011). A qualitative study of performance leadership and management in elite sport. Journal of Applied Sport Psychology, 23, 223-242. 
Ghaye, T., \& Lillyman, S. (2000). Reflection: Principles and practice for healthcare professionals. Wiltshire, UK: Quay Books.

Kim, W. Chan., \& Mauborgne, R. (2003). Tipping point leadership. Harvard Business Review, 81(4), 60-69.

League Managers Association (2010). LMA end of season statistics 2010. Retrieved from http://www.leaguemanagers.com/news/news-6585.html

Martindale, A., \& Collins, D. (2007). Enhancing the evaluation of effectiveness with professional judgment and decision making. The Sport Psychologist, 21, 458-474.

Zinser, L. (2008, December 17). One loss away from unemployment. The New York Times. Retrieved from http://www.nytimes.com/2008/12/18/sports/18coaches. 